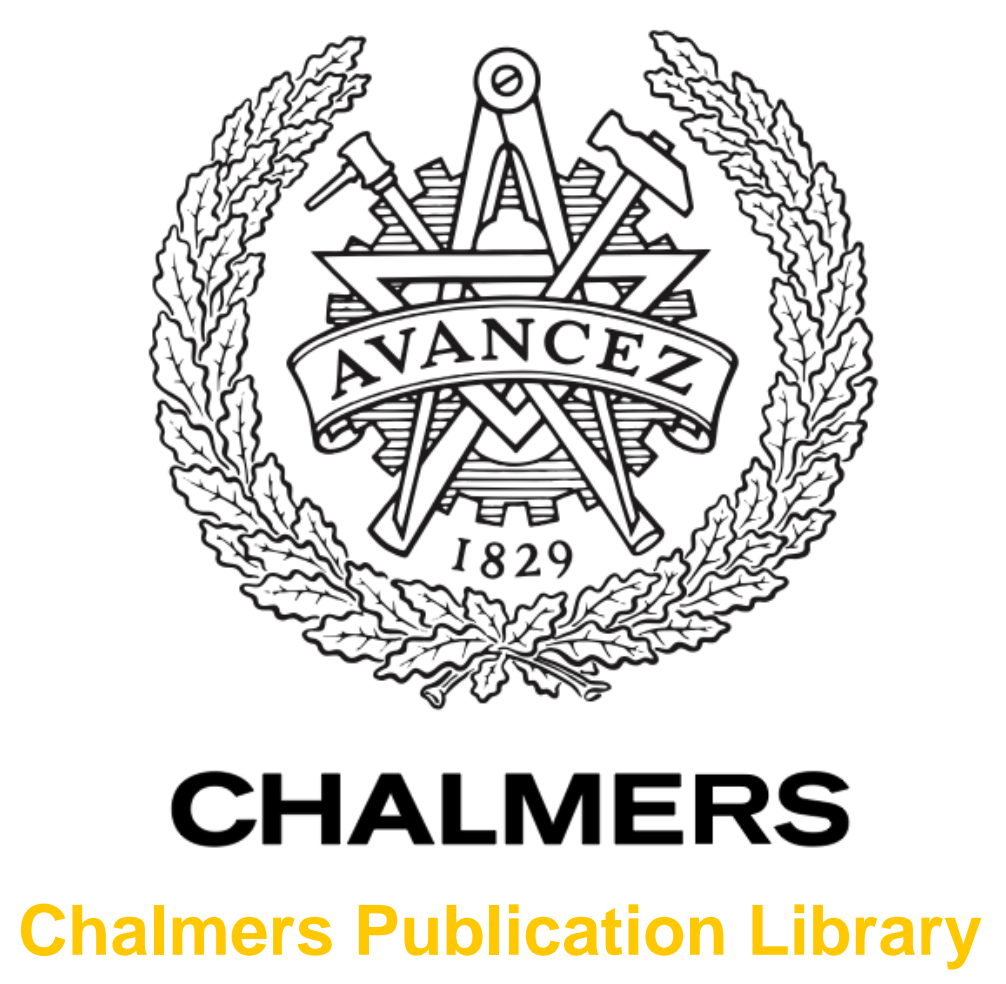

\title{
GaN nanorod light emitting diodes with suspended graphene transparent electrodes grown by rapid chemical vapor deposition
}

This document has been downloaded from Chalmers Publication Library (CPL). It is the author's version of a work that was accepted for publication in:

Applied Physics Letters (ISSN: 0003-6951)

Citation for the published paper:

Xu, K. ; Xu, C. ; Xie, Y. (2013) "GaN nanorod light emitting diodes with suspended graphene transparent electrodes grown by rapid chemical vapor deposition". Applied Physics Letters, vol. 103(22), pp. 5.

http://dx.doi.org/10.1063/1.4836375

Downloaded from: http://publications.lib.chalmers.se/publication/192036

Notice: Changes introduced as a result of publishing processes such as copy-editing and formatting may not be reflected in this document. For a definitive version of this work, please refer to the published source. Please note that access to the published version might require a subscription. 


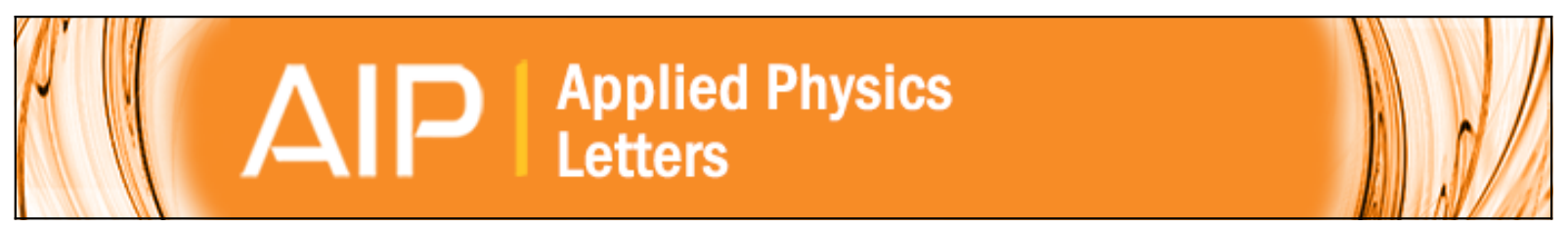

GaN nanorod light emitting diodes with suspended graphene transparent electrodes grown by rapid chemical vapor deposition

Kun Xu, Chen Xu, Yiyang Xie, Jun Deng, Yanxu Zhu, Weiling Guo, Mingming Mao, Meng Xun, Maoxing Chen, Lei Zheng, and Jie Sun

Citation: Applied Physics Letters 103, 222105 (2013); doi: 10.1063/1.4836375

View online: http://dx.doi.org/10.1063/1.4836375

View Table of Contents: http://scitation.aip.org/content/aip/journal/apl/103/22?ver=pdfcov

Published by the AIP Publishing

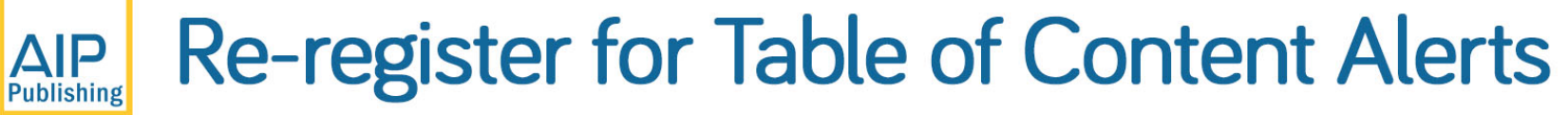




\title{
GaN nanorod light emitting diodes with suspended graphene transparent electrodes grown by rapid chemical vapor deposition
}

\author{
Kun Xu, ${ }^{1}$ Chen Xu, ${ }^{1, a)}$ Yiyang Xie, ${ }^{2}$ Jun Deng, ${ }^{1}$ Yanxu Zhu, ${ }^{1}$ Weiling Guo, ${ }^{1}$ Mingming Mao, ${ }^{1}$ \\ Meng Xun, ${ }^{1}$ Maoxing Chen, ${ }^{1}$ Lei Zheng, ${ }^{1}$ and Jie Sun ${ }^{1,3, b)}$ \\ ${ }^{1}$ Key Laboratory of Optoelectronics Technology, Beijing University of Technology, Ministry of Education, \\ Beijing 100124, China \\ ${ }^{2}$ State Key Laboratory of Integrated Optoelectronics, Institute of Semiconductor, \\ Chinese Academy of Sciences, Beijing 100083, China \\ ${ }^{3}$ Mikroteknologi och Nanovetenskap, Chalmers Tekniska Högskola AB, Göteborg 41296, Sweden
}

(Received 22 September 2013; accepted 12 November 2013; published online 26 November 2013)

\begin{abstract}
Ordered and dense GaN light emitting nanorods are studied with polycrystalline graphene grown by rapid chemical vapor deposition as suspended transparent electrodes. As the substitute of indium tin oxide, the graphene avoids complex processing to fill up the gaps between nanorods and subsequent surface flattening and offers high conductivity to improve the carrier injection. The asfabricated devices have $32 \%$ improvement in light output power compared to conventional planar $\mathrm{GaN}$-graphene diodes. The suspended graphene remains electrically stable up to $300^{\circ} \mathrm{C}$ in air. The graphene can be obtained at low cost and high efficiency, indicating its high potential in future applications. @ 2013 AIP Publishing LLC. [http://dx.doi.org/10.1063/1.4836375]
\end{abstract}

GaN compounds are widely used to fabricate light emitting diodes (LEDs) covering the spectrum from yellow to ultraviolet. GaN and related materials are major players in the industry of high light intensity blue, green, and violet LEDs and lasers. GaN LEDs have attracted extensive interest also due to their tolerance of high temperature, radiation, and corrosive environment. However, due to its high refractive index, photons generated by active regions can be totally reflected at the GaN/air interface easily. ${ }^{1,2}$ The critical angle for light escape cone is $\sim 23^{\circ}$ (GaN refractive index $n=2.5$ ), rendering the extraction of only $4 \%$ of the total internal light. ${ }^{1}$ Also, the lattice mismatch between $\mathrm{In}(\mathrm{Ga}) \mathrm{N}$ and $\mathrm{GaN}$ creates a large strain, which in turn leads to high piezoelectric field and hence quantum-confined Stark effect. In order to improve the quantum efficiency of $\mathrm{GaN}$ LEDs, nanorods have been proposed, where the conventional planar architecture is changed into onedimensional nanostructures. It turns out to be an efficient method to address these problems, mainly due to its super large light emitting areas, optical waveguide effect, and released strains. ${ }^{3-5}$ However, the device fabrication in $\mathrm{GaN}$ nanorod LEDs is complicated. The length of the dry-etched nanorods should be higher than the depth of active region in the device to form fully separated nanorods, which unavoidably makes $p$-GaN discontinuous. Considering that even in traditional continuous $p$-GaN layer, because of its low conductivity caused by difficulties in $p$-doping, an extra transparent conducting layer is needed to spread the current laterally, this transparent electrode is indeed indispensable for nanorod LEDs to ensure a proper carrier injection into all the active regions. Typically, insulation mediums such as polymers or spin-on glass are used to fill the gaps between nanorods to avoid contact of the transparent electrode to $n$-GaN or active layers. ${ }^{3,5}$ The process is rather complex as

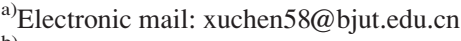

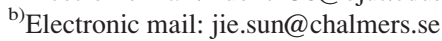

it generally requires additional steps of dry etching or chemical mechanical polishing to obtain a flat, clean, and fresh top surface of the nanorods for $p$-electrode contact.

Another issue is that the typical material for transparent electrodes, indium tin oxide (ITO), has an increasingly high price as indium is slathered and getting scarce. Also, ITO is nontransparent at low wavelength regimes, which is not suitable for ultraviolet GaN LEDs. Graphene, a truly two dimension material with hexagonal $s p^{2}$ hybridized carbon lattice, is transparent, conductive, and flexible. The addition of one layer of graphene loses merely $2.3 \%$ of transparency. The optical transmittance remains very high from infrared to ultraviolet frequencies. Recently, it can be grown by a semiconductor industry compatible technique chemical vapor deposition (CVD) in large area at relatively low cost. ${ }^{6-9}$ Therefore, it has a high potential in the fabrication of transparent conducting layer instead of ITO. Nevertheless, the conductivity of graphene film is yet generally lower than that of ITO (the sheet resistance of typical monolayer CVD graphene is in $\mathrm{k} \Omega$ range) even though under well optimized conditions it outperforms the latter. ${ }^{6}$ There are many attempts to improve the conductivity of graphene, mostly by doping, but unfortunately one has to somehow sacrifice the transparency, ${ }^{6,10,11}$ and the doping effect is hardly stable. ${ }^{12}$ On the other hand, it is known that the carrier mobility in suspended graphene is much higher than graphene on substrates, ${ }^{13}$ by virtue of the reduced charge impurity scattering and so on. Indeed, Rouhi et al. ${ }^{14}$ have measured the sheet resistance of suspended few layer graphene grown on Ni by CVD, and the result is as low as $10 \Omega / \square$. Note the commercial ITO film applied in GaN LEDs has a $R_{S}$ ranging from 10 to $50 \Omega / \square$ typically. To date, many publications report suspended graphene in electronic devices, ${ }^{15,16}$ but there are rarely any literatures on making use of the high conductivity of suspended graphene in optoelectronic devices.

In this letter, three layers of graphene successively grown by CVD are employed as transparent electrodes in 
GaN nanorod LEDs. The nanorods are well ordered resembling photonic crystals, and the gaps in between are optimized to obtain better device performances. The graphene is transferred directly onto GaN nanorods and remains suspended by virtue of its excellent mechanical strength, so there is no need to fill the gap between nanorods with insulators, which greatly simplifies the device processing. The transparency is maintained because the as-synthesized graphene has not undergone any deteriorative processing. The graphene suspended between nanorods is highly conductive and solves the current crowding problem. Furthermore, the electrical property of the graphene on nanorods is very stable. Previously, contrary to many groups pursuing large graphene single crystals at slow deposition rates, we demonstrated the so-called rapid CVD growth of polycrystalline graphene with superior optical and electrical properties, which is a technology oriented towards scalable, time- and cost-effective industrial applications. ${ }^{9}$ In the continuation work here, the high mechanical property of such graphene is also confirmed. Our experiment offers a promising pathway for the utilization of fast CVD grown graphene as transparent electrodes in GaN-based optoelectronics, potentially replacing ITO in industrial applications.

Commercial blue LED wafers with $c$-plane sapphire substrates are used in our experiment. The epitaxial structures, from bottom to top, are in turn $2 \mu \mathrm{m}$ undoped $(u)-\mathrm{GaN}$, $2 \mu \mathrm{m} n$-GaN, about $100 \mathrm{~nm} \mathrm{InGaN} \mathrm{multiple} \mathrm{quantum} \mathrm{wells}$ (MQWs) and $150 \mathrm{~nm} p$-GaN. The size of LED devices is $10 \mathrm{mil} \times 16 \mathrm{mil}(1 \mathrm{mil}=25.4 \mu \mathrm{m})$. The nanorod processing is as follows. One layer of hexagonally ordered, closely packed $\mathrm{SiO}_{2}$ nanospheres with diameter $580 \mathrm{~nm}$ is made by dip-coating deposition method. In brief, the nanospheres are dispersed in a solution where they form a monolayer of spheres on the surface of liquid. Then, the GaN wafer is dipped into the solution and pulled out with a controlled speed and tilt angle. Afterwards, the GaN epi-wafer is dry-etched for $22 \mathrm{~s}$ by inductively coupled plasma (ICP) to form nanorods, using the ordered $\mathrm{SiO}_{2}$ nanospheres as etching mask, in $40 \mathrm{sccm} \mathrm{Cl}_{2}$ and $5 \mathrm{sccm} \mathrm{SiCl}_{4}$. The wafer is organically cleaned, followed by HF solution treatment to remove the residual $\mathrm{SiO}_{2}$ nanospheres and the native oxide layer of $\mathrm{GaN}$. The graphene is grown by CVD on $\mathrm{Cu}$ foils, and the details are published elsewhere. ${ }^{9,17,18} \mathrm{~A}$ unique feature of our graphene is that it is grown rapidly in merely $5 \mathrm{~min}$, by virtue of our special design of the system and experimental conditions. For real applications, this is desirable because the cost on energy and time will be drastically reduced. Despite the high nucleation density induced polycrystalline nature, the graphene shows good optical and electrical properties. ${ }^{9,17,18}$ The transparency of three layer graphene is approximately the same as $240 \mathrm{~nm}$ ITO in visible light region. ${ }^{9}$ The sheet resistances $R_{S}$ of the mono- and tri-layer graphene are $\sim 3000 \Omega / \square$ and $\sim 1000 \Omega / \square$, respectively. Thereinafter, we will demonstrate that the mechanical characteristics is also as good as standard graphene. Monolayer graphene is wet transferre ${ }^{18}$ to the GaN nanorods, and the procedure is repeated three times to obtain 3-layer graphene. After each transfer, the sample is washed in $\mathrm{H}_{2} \mathrm{O}$ and gently blown dry by $\mathrm{N}_{2}$ without using any sophisticated techniques such as critical point drying. In short, poly(methyl methacrylate) is spun on the graphene/Cu and immersed in concentrated $\mathrm{FeCl}_{3}$ solution to etch $\mathrm{Cu}$. Then, the graphene on polymer is cleaned and placed on the GaN nanorod surface. Finally, the polymer is dissolved in acetone. The suspended graphene attaches well to the top of nanorods through Van der Waals bonding. The LEDs are fabricated by two steps of photolithography. In the first patterning, mesas are defined by ICP etching to $1.1 \mu \mathrm{m}$ deep to reach the heavily doped $n$-GaN layer. The suspended 3-layer graphene is patterned and etched simultaneously. Second, Ti/Au $(15 \mathrm{~nm} / 100 \mathrm{~nm}) p$ and $n$ metal

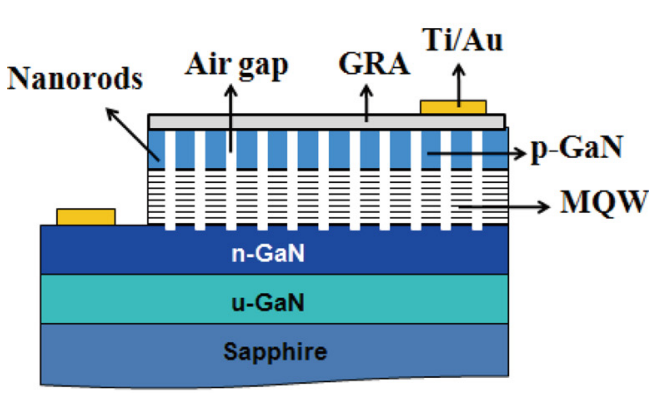

(a)

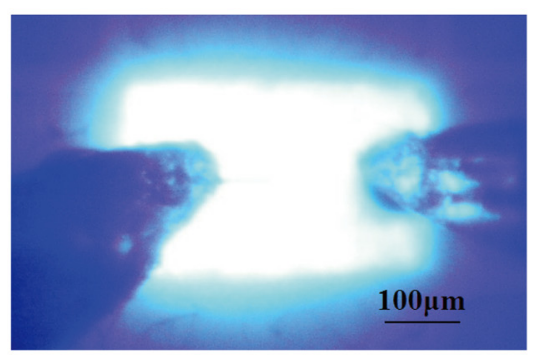

(c)

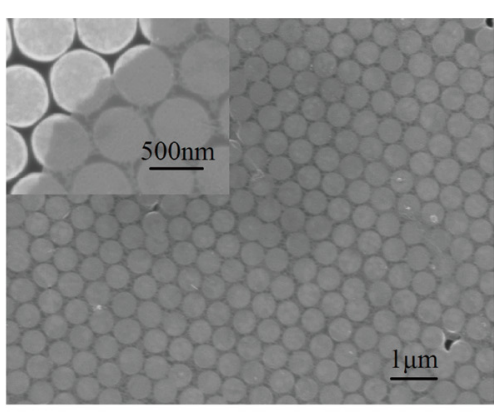

(b)
FIG. 1. (a) Schematic diagram of the GaN nanorod LED structures, where the length of nanorods is etched to be slightly deeper than the multiple quantum wells. Three-layer graphene (denoted as GRA) is suspended and used as transparent electrodes. (b) SEM micrographs of the graphene on nanorods. The inset is a zoom-in image which is taken at a place where the graphene partly covers the rods for easy identification of the suspended graphene. (c) Photo of the electroluminescence from GaN nanorod LEDs. Evidently, the graphene film on nanorods affords a good current spreading effect. 


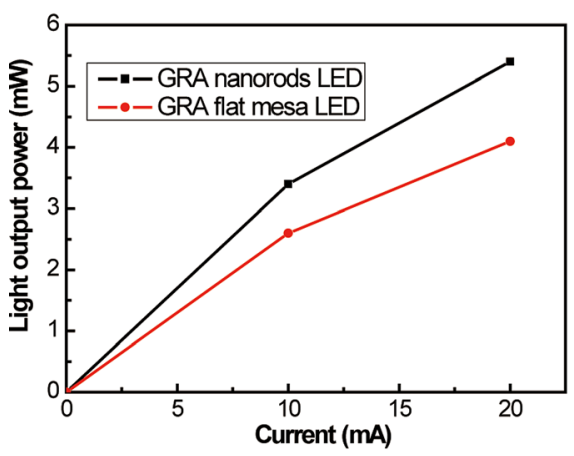

(a)

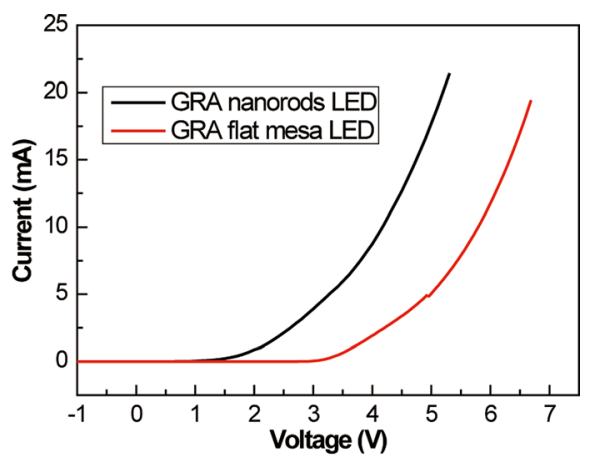

(b) electrodes are fabricated by lift-off lithography and sputtering. The device schematic diagram of the GaN nanorod LEDs can be found in Fig. 1(a).

Fig. 1(b) shows a scanning electron microscopy (SEM) image of the suspended graphene on $\mathrm{GaN}$ nanorods with an average diameter of $520 \mathrm{~nm}$. It can be seen that the nanorods are very dense and well ordered, in contrast with those irregular rods from self-organized bottom-up approaches or by etching with randomly deposited nanosphere masks. For every mesa, we try to squeeze in many nanorods to enlarge the light emitting surface while yet keeping each rod independent. This layout is controllable and reproducible. The inset of Fig. 1(b) shows an SEM micrograph with an uncovered area on the nanorods, which generates some contrast and makes the suspended graphene more visible. The graphene appears to be mechanically strong and robust, for its survival of all the lithographic steps and the gentle blow dry in $\mathrm{N}_{2}$. The photo of Fig. 1(c) shows uniform electroluminescence of a GaN-graphene LED at inject current $I=20 \mathrm{~mA}$, indicating the effective current spreading on nanorods. The high mechanical strength of the graphene film ensures that it can protect the $n$-GaN and active layers from contacting the $p$-pad and short out the $p$ - $n$ junction. Therefore, this technique does not require the filling of gaps between the nanorods, which is much simpler than the case for ITO. A batch of control devices of GaN LEDs using the same type of epitaxial wafers are also fabricated, where they have traditional mesas with flat tops. Again, three layers of graphene are transferred onto the plane mesa serving as the transparent conductive layer. Fig. 2 provides an optoelectronic property comparison of the GaN nanorod LEDs with suspended graphene electrodes and the traditional plane mesa GaN-graphene LEDs. Fig. 2(a) plots the electroluminescent light output power vs. current. At $I=20 \mathrm{~mA}$, the light output power of the nanorod LEDs is improved by $32 \%$ as compared with the flat mesa counterparts. The effect can be attributed to both the utilization of nanorod structure and the suspension-induced enhancement of the graphene conductivity. Nanorods lead to super large light emitting areas, optical waveguide effect, and released strains, improving the quantum efficiency. A well conducting graphene layer can enhance the carrier injection of the nanorods that are relatively far away from the $p$-electrodes (metal), reducing the otherwise severe current crowding and local overheat in the LEDs. Fig. 2(b) plots the $I$ - $V$ curves of the two types of devices, showing typical diode behaviors.
Previously, we suggested an interlayer technology to bridge the Fermi levels of the $p-\mathrm{GaN}$ and graphene in order to lower the potential barrier at the contact. ${ }^{9}$ Here, however, it is not implemented for simplicity. The specific contact resistance $\rho_{c}$ of our $\mathrm{Au} / \mathrm{Ti} / \mathrm{graphene} / \mathrm{p}-\mathrm{GaN}$ junction is $0.8-1.8 \Omega \cdot \mathrm{cm}^{2}$ as measured by transmission line model (TLM) method. The values are in line with literature. ${ }^{10}$ In Fig. 2(b), the forward voltage $V$ of the nanorod LED is smaller than that of plane mesa LED, hinting that possibly a parallel leakage current path exists on the nanorod surfaces caused by ICP etching induced defects. This phenomenon is typically observed in conventional GaN nanorod LEDs with ITO as well ${ }^{3,19}$ and is not related to graphene. Calculated from Fig. 2, the wall-plug efficiency (ratio between optical power and electrical power) of the GaN-graphene nanorod device is $5.2 \%$ at $I=20 \mathrm{~mA}$, an order of magnitude higher than that of GaN nanorod LEDs with $\mathrm{Ni} / \mathrm{Au}$ transparent electrodes. ${ }^{20}$ At this stage, however, it is still several times smaller compared to ITO-based GaN nanorod LEDs, mainly due to the yet resistive graphene and its contact to $\mathrm{GaN}$. As this problem is not intrinsic, with future breakthroughs in graphene doping/Fermi level engineering, the strategy in this letter is expected to afford a viable pathway towards LEDs outperforming conventional ITO-based devices.

In order to systematically investigate the conductivity of the suspended graphene, we design the structure shown in Fig. 3(a) to measure the $I-V$ characteristics of the graphene film both on $\mathrm{SiO}_{2}$ nanorods (the layout and diameter of the nanorods are exactly the same as those of the $\mathrm{GaN}$ nanorods used in the devices) and planar $\mathrm{SiO}_{2}$ substrate. We opt to use insulating $\mathrm{SiO}_{2}$ test structures instead of GaN simply because the finite conductance from $p-\mathrm{GaN}$ beneath the graphene will affect the electrical measurements otherwise. Estimating from Fig. 3(b), the sheet resistance of graphene on plane substrate is 3.3 times as large as that on nanorods $(1000 \pm 100 \Omega / \square$ vs. $300 \pm 30 \Omega / \square$ ). Here, Ti/Au is known to provide the lowest contact resistivity to graphene. ${ }^{21}$ Furthermore, our structures are annealed in $\mathrm{N}_{2}$ atmosphere for $2 \mathrm{~min}$ at $300^{\circ} \mathrm{C}$ and, therefore, we neglect the contact resistance in the calculation for simplicity. The improvement in conductivity is mainly due to the fact that the suspended graphene between nanorods has much higher carrier mobility by virtue of less charge impurity scattering during transport. ${ }^{16}$ As shown previously, this effect has contributed to the higher light output power of the nanorod LEDs with respect to their flat mesa counterpart. In 


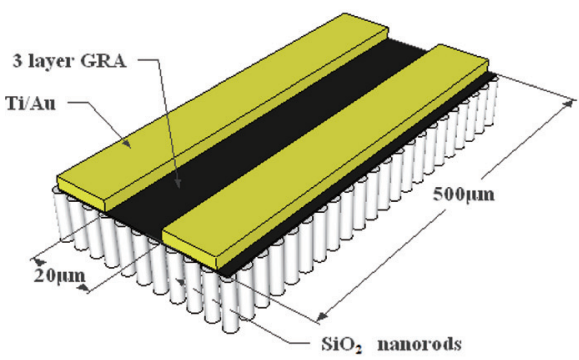

(a)

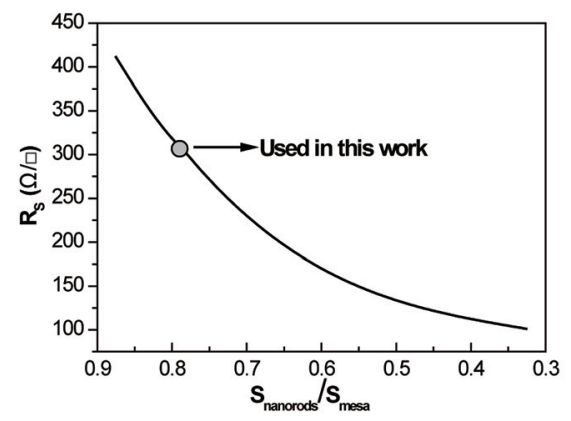

(c)

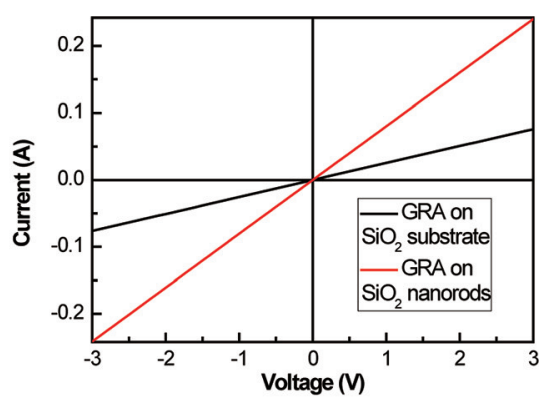

(b)
FIG. 3. (a) Structure used to measure graphene sheet resistance $R_{s}$ on $\mathrm{SiO}_{2}$ nanorods, where the three-layer graphene are contacted by two Ti/Au $(15 \mathrm{~nm} / 100 \mathrm{~nm})$ pads. (b) Comparison of the $I-V$ curves of the tri-layer graphene on nanorod $\mathrm{SiO}_{2}$ and on flat $\mathrm{SiO}_{2}$ substrate, showing an improvement in $R_{s}$ by a factor of 3.3 due to the suspension reduced scattering. (c) Curve showing the relationship between the graphene overall sheet resistance and the $\mathrm{SiO}_{2}$ nanorod area occupation. These data help to seek the optimal $\mathrm{GaN}$ nanorod diameter for the most effective light emitting. GRA denotes graphene.
Fig. 3(a), based on the measurement above, the $R_{s}$ for the graphene atop nanorods is $\sim 1000 \Omega / \square$ whereas the overall $R_{s}$ is $\sim 300 \Omega / \square$. Using finite element analysis, the $R_{s}$ for the purely freestanding graphene part is self-consistently calculated to be $\sim 50 \Omega / \square$, which is about 20 times as large as that of graphene on $\mathrm{SiO}_{2}$ substrate, similar to what other groups have found. ${ }^{14}$ Obviously, shrinking the size of nanorods will enlarge the freestanding areas of graphene and enhance the electrical interconnect among nanorods. Fig. 3(c) shows the calculated relationship between the graphene average sheet resistance $R_{S}$ and the area ratio of the nanorods in the whole mesa. The resistance decreases sharply as the ratio goes down. Nevertheless, if the freestanding portion of the graphene is too large, the lighting surface will be reduced and hence lower the output power. As the result of tradeoff, ratio 0.78 is used for the GaN nanorod LEDs in this letter.

Device reliability is vital for real applications. To estimate the stability of suspended graphene formed on nanorods, high temperature treatment is carried out. The test structures used are again graphene on nanorod and planar $\mathrm{SiO}_{2}$ with the same device geometry as Fig. 3(a). The samples are both baked in open air for various time and temperatures, and the results are summarized in Table I. It is clear that the conductivity of graphene on nanorods is very stable up to $300^{\circ} \mathrm{C}$ in air. The same antioxidation behavior is observed in the graphene on planar $\mathrm{SiO}_{2}$ substrate. An increase in $R_{S}$ is only discovered when $T$ increases to $400{ }^{\circ} \mathrm{C}$. In this case, the degradation of the graphene on nanorods is much more severe, for the fact that $R_{S}$ increases by an order of magnitude while only twice for the graphene on $\mathrm{SiO}_{2}$ plane. The difference can be explained by the more exposed areas in the suspended graphene structure which are easier to oxidize. Considering that optoelectronic devices generally have work temperatures well below $200^{\circ} \mathrm{C}$, most LEDs even under $100^{\circ} \mathrm{C}$, the high conductivity of the suspended graphene is hereby confirmed to be stable for applications.

Summarily, polycrystalline graphene films are grown with $\mathrm{Cu}$ catalyst by fast CVD technology. Three layers of graphene are transferred to well ordered and densely packed GaN nanorod LED structures as the suspended transparent electrodes. At least three key roles of graphene have been realized in this study, namely, (1) avoiding the complex processing to fill up the gaps between nanorods and the subsequent surface flattening; (2) replacing ITO and addressing the issues with indium scarcity and narrow spectrum in transmittance; (3) enhancing the graphene conductivity so that the carrier injection is boosted. Uniform electroluminescence is achieved in such devices, where the light output power of the nanorod LEDs with graphene electrodes improves $32 \%$ as compared to conventional planar GaN-graphene LEDs. At the nanorod diameter of $520 \mathrm{~nm}$, the free-standing graphene between nanorods is $\sim 20$ times as conductive as attached to $\mathrm{SiO}_{2}$ substrate, resulting in the 3.3 times lower overall $R_{s}$. The high conductivity from the suspended graphene remains

TABLE I. Graphene sheet resistances under different baking conditions in open air.

\begin{tabular}{lccc}
\hline \hline & & \multicolumn{2}{c}{ Sheet resistance $\left(R_{s}, \Omega / \square\right)$} \\
\cline { 3 - 4 } $\begin{array}{l}\text { Baking } \\
\text { time } t(\mathrm{~h})\end{array}$ & $\begin{array}{c}\text { Baking } \\
\text { temperature } T\left({ }^{\circ} \mathrm{C}\right)\end{array}$ & $\begin{array}{c}\text { Graphene on } \\
\text { nanorod } \mathrm{SiO}_{2}\end{array}$ & $\begin{array}{c}\text { Graphene on } \\
\text { planar } \mathrm{SiO}_{2}\end{array}$ \\
\hline 2 & 100 & 311 & 999 \\
4 & 200 & 316 & 1064 \\
6 & 300 & 298 & 1060 \\
7 & 400 & 4021 & 2035 \\
\hline \hline
\end{tabular}


stable up to $300^{\circ} \mathrm{C}$ in air. This work confirms that the graphene grown by rapid CVD has equally high optical, electrical and mechanical properties compared with standard graphene, but can be obtained at much lower cost and higher efficiency, hinting its promising future in real life applications. Finally, we note that the suspended graphene technology developed in this letter should not be limited to LEDs, and it is expected to contribute to other related structures such as nanowire devices, solar cells as well.

This work was supported by the National Natural Science Foundation of China (Nos. 61376049 61076044, 61107026, and 61204011), the Natural Science Foundation of Beijing, China (Nos. 4132006, 4102003, and 4112006), the Specialized Research Fund for the Doctoral Program of Higher Education of China (No. 20121103110018), the National High Technology Research and Development Program of China (Nos. 2013AA031903 and 2008AA03Z402), and the Special Grant for BJUT Large-Area Layer-Controllable Graphene Carbon Nanoelectronics Teaching and Research Base.

${ }^{1}$ T. Fujii, Y. Gao, R. Sharma, E. L. Hu, S. P. DenBaars, and S. Nakamura, Appl. Phys. Lett. 84, 855 (2004).

${ }^{2}$ F. A. Ponce and D. P. Bour, Nature 386, 351 (1997).

${ }^{3}$ M.-Y. Ke, C.-Y. Wang, L.-Y. Chen, H.-H. Chen, H.-L. Chiang, Y.-W. Cheng, M.-Y. Hsieh, C.-P. Chen, and J. Huang, IEEE J. Sel. Top. Quantum Electron. 15, 1242 (2009).

${ }^{4}$ J. H. Son, J. U. Kim, Y. H. Song, B. J. Kim, C. J. Ryu, and J. L. Lee, Adv. Mater. 24, 2259 (2012).

${ }^{5}$ L. Y. Chen, Y. Y. Huang, C. H. Chang, Y. H. Sun, Y. W. Cheng, M. Y. Ke, C. P. Chen, and J. J. Huang, Opt. Express 18, 7664 (2010).
${ }^{6}$ S. Bae, H. Kim, Y. Lee, X. F. Xu, J. S. Park, Y. Zheng, J. Balakrishnan, T. Lei, H. R. Kim, Y. I. Song, Y. J. Kim, K. S. Kim, B. Ozyilmaz, J. H. Ahn, B. H. Hong, and S. Iijima, Nat. Nanotechnol. 5, 574 (2010).

${ }^{7}$ W. W. Cai, Y. W. Zhu, X. S. Li, R. D. Piner, and R. S. Ruoff, Appl. Phys. Lett. 95, 123115 (2009).

${ }^{8}$ H. Cao, Q. Yu, L. A. Jauregui, J. Tian, W. Wu, Z. Liu, R. Jalilian, D. K. Benjamin, Z. Jiang, J. Bao, S. S. Pei, and Y. P. Chen, Appl. Phys. Lett. 96, 122106 (2010).

${ }^{9}$ K. Xu, C. Xu, J. Deng, Y. X. Zhu, W. L. Guo, M. M. Mao, L. Zheng, and J. Sun, Appl. Phys. Lett. 102, 162102 (2013).

${ }^{10}$ S. Chandramohan, J. H. Kang, Y. S. Katharria, N. Han, Y. S. Beak, K. B. Ko, J. B. Park, H. K. Kim, E. K. Suh, and C. H. Hong, Appl. Phys. Lett. 100, 023502 (2012).

${ }^{11}$ I. Khrapach, F. Withers, T. H. Bointon, D. K. Polyushkin, W. L. Barnes, S. Russo, and M. F. Craciun, Adv. Mater. 24, 2844 (2012).

${ }^{12}$ K. K. Kim, A. Reina, Y. M. Shi, H. Park, L. J. Li, Y. H. Lee, and J. Kong, Nanotechnology 21, 285205 (2010).

${ }^{13}$ K. I. Bolotin, K. J. Sikes, Z. Jiang, M. Klima, G. Fudenberg, J. Hone, P. Kim, and H. L. Stormer, Solid State Commun. 146, 351 (2008).

${ }^{14}$ N. Rouhi, Y. Y. Wang, and P. J. Burke, Appl. Phys. Lett. 101, 263101 (2012).

${ }^{15}$ M. T. Allen, J. Martin, and A. Yacoby, Nature Commun. 3, 934 (2012).

${ }^{16} \mathrm{~N}$. Tombros, A. Veligura, J. Junesch, M. H. D. Guimaraes, I. J. VeraMarun, H. T. Jonkman, and B. J. van Wees, Nat. Phys. 7, 697 (2011).

${ }^{17}$ J. Sun, N. Lindvall, M. T. Cole, K. T. T. Angel, T. Wang, K. B. K. Teo, D. H. C. Chua, J. Liu, and A. Yurgens, IEEE Trans. Nanotechnol. 11, 255 (2012).

${ }^{18}$ C. J. L. de la Rosa, J. Sun, N. Lindvall, M. T. Cole, Y. Nam, M. Löffler, E. Olsson, K. B. K. Teo, and A. Yurgens, Appl. Phys. Lett. 102, 022101 (2013).

${ }^{19}$ M. Y. Hsieh, C. Y. Wang, L. Y. Chen, M. Y. Ke, and J. J. Huang, IEEE J. Quantum Electron. 44, 468 (2008).

${ }^{20}$ H.-M. Kim, Y.-H. Cho, H. Lee, S. I. Kim, S. R. Ryu, D. Y. Kim, T. W. Kang, and K. S. Chung, Nano Lett. 4, 1059 (2004).

${ }^{21}$ J. A. Robinson, M. LaBella, M. Zhu, M. Hollander, R. Kasarda, Z. Hughes, K. Trumbull, R. Cavalero, and D. Snyder, Appl. Phys. Lett. 98, 053103 (2011). 